\title{
Identifying an Important Ingredient for Safe Discharge to Home Oxygen Therapy: The Respiratory Therapist and the RIsOTTO Study
}

COPD is a leading cause of death around the world and imparts considerable morbidity and health care costs. Exacerbations requiring hospitalization are common, and, at least in the United States, patients are usually discharged from the hospital after clinical improvement but typically have not returned to baseline physiologic status. Thus, patients who have not been on oxygen at home may need oxygen upon discharge at least in the short-term, and those previously on oxygen may need a change in their prescription. Oxygen can be prescribed continuously with or without ambulatory oxygen, with exertion only, or at night. ${ }^{1}$ Ideally, the patient's oxygen needs should be evaluated prior to discharge and appropriate supplemental oxygen ordered. However, a recent retrospective study of subjects with COPD revealed that, while documentation of resting hypoxemia was good, evaluation for exertional hypoxemia was usually lacking. ${ }^{2}$ Furthermore, documentation that prescribed oxygen was adequate during both rest and with exertion was also typically lacking. Hospitalists are extremely busy and often do not have control over their time as they have to address patients' acute needs. A more systematic approach with use of experienced nonphysicians such as respiratory therapists might lead to better results with respect to oxygen prescriptions. Usually nursing, pharmacy, and social work staff participate in discharge planning, but this help does not extend to oxygen supplementation.

In the study by Tan et $\mathrm{al}^{3}$ in this issue of RESPIRATORY CARE, the authors surveyed hospital-based respiratory therapists who were members of the American Association for Respiratory Care to ask how often they evaluated the need for home oxygen in patients admitted with an exacerbation of COPD. There were 611 respondents, of whom 490 met entry criteria and were included in the analysis. To put this response in perspective, there are an estimated 170,000 respiratory therapists working in the United States. Nevertheless, the number who said they consistently (defined as $>75 \%$ ) made such evaluations was $58 \%$

\footnotetext{
The author has disclosed no conflicts of interest.

Correspondence: M Jeffery Mador MD, Western New York VA Healthcare System, Division of Pulmonary, Critical Care and Sleep Medicine, 3495 Bailey Ave, Buffalo NY 14215. E-mail:mador@buffalo.edu.
}

DOI: $10.4187 /$ respcare. 08832 for resting hypoxemia, $43 \%$ for exertional hypoxemia, and $14 \%$ for nocturnal hypoxemia. Only $25 \%$ of respiratory therapists were consistently involved in the decisionmaking for the home oxygen equipment the patient

\section{See the Original Study on Page 183}

would receive. Patients being discharged from the hospital after an exacerbation of COPD are rarely discharged without oxygen if they demonstrate resting hypoxemia meeting Medicare criteria for oxygen supplementation. Documentation that prescribed oxygen is adequate at rest is also usually present because oxygen saturation is recorded with the vital signs and these values are usually easily obtained from the electronic medical record, although the level of supplemental oxygen may be less carefully documented. However, assessment for exertional hypoxemia is usually not done as outlined in the retrospective review by Zaidi et $\mathrm{al}^{2}{ }^{2}$ nor is an assessment of levels of supplemental oxygen required to prevent exertional hypoxemia. Assessment for nocturnal hypoxemia is rarely done prior to discharge.

The first question is whether treatment of exertional hypoxemia or nocturnal hypoxemia alters outcomes in patients with COPD. If it doesn't, there is little point in assessing patients for these intermittent forms of hypoxemia. The American Thoracic Society (ATS) recently addressed this issue after reviewing the evidence to support the use of ambulatory oxygen in patients with and without resting hypoxemia. ${ }^{4}$ The primary outcomes they addressed were quality of life and exercise performance. They found small inconsistent improvement in dyspnea (below the minimally important clinical difference) that generally favored treatment and improvement in laboratory exercise outcomes. Quality of life in patients who also had resting hypoxemia has not been adequately studied. The ATS conditionally recommended (ie, with low-quality evidence) ambulatory oxygen while recognizing that more research is required to address the effects of ambulatory oxygen on patient-centered outcomes (eg, dyspnea, quality of life, fatigue, physical activity). Data on treating nocturnal hypoxemia are even more limited. However, a recent study and meta-analysis concluded that nocturnal oxygen had no effect on mortality, progression to continuous oxygen, 


\section{EDITORIALS}

exacerbations, or hospitalizations..$^{5}$ Effects on quality of life have not been assessed. In the Long-term Oxygen Treatment Trial (LOTT), subjects who desaturated during exertion were treated with nocturnal and ambulatory oxygen and had no difference in quality of life or 6-min walk distance compared to a control group; however, oximetry to document nocturnal hypoxemia was not performed in this study. ${ }^{6}$

Thus, if we accept the current ATS recommendations, which are certainly not universally accepted, then it is reasonable to supply ambulatory oxygen to patients with COPD after exacerbation. If that is the case, then data suggest there is room for improvement in how we assess exertional requirements and adequacy of exertional oxygen prescription. Having a respiratory therapist routinely assess patients admitted with an exacerbation of COPD prior to discharge for both resting and exertional oxygen requirements is a potential solution to this problem; the survey data suggest it is already being done in many institutions. ${ }^{3}$ Since only $42 \%$ of surveyed respiratory therapists stated they were very familiar with Centers for Medicare and Medicaid Services guidelines for home oxygen, additional education would be required.

Finally, the survey showed that respiratory therapists are not usually involved in the choice of home oxygen equipment that the patient receives upon discharge. The survey did not address education of patients on their home equipment, which is usually provided by the durable medical equipment provider. In treatment of sleep apnea, respiratory therapists either take the lead or collaborate with the physician in decisions about mask type and CPAP troubleshooting. They also provide the bulk of patient teaching. Further research into whether a similar approach to ambulatory oxygen might lead to better patient experiences and greater utilization of ambulatory oxygen would be informative. Some of the current disparate results with ambulatory oxygen are due in part to suboptimal usage, which in turn may be due to incomplete patient education and choices for portable oxygen that do not reflect each patient's needs.

M Jeffery Mador

Division of Pulmonary, Critical Care and Sleep Medicine

University of Buffalo

Buffalo, New York

\section{REFERENCES}

1. Savani R, Patil M, Tariq A, Mador MJ. A retrospective observational study of domiciliary oxygen usage in a subset of veterans. Respir Care 2020;65(11):1694-1701.

2. Zaidi F, Lee RS, Buchcic BA, Bracken NE, Jaffe HA, Joo M, et al. Evaluation and documentation of supplemental oxygen requirements is rarely performed in patients hospitalized with COPD. Chronic Obstr Pulm Dis 2017;4(4):287-296.

3. Tan AM, Vines DL, Krishnan JA, Prieto-Centurion V, Kallstrom TJ. Home oxygen evaluation by respiratory therapists in patients hospitalized for COPD exacerbation: the RIsOTTO study. Respir Care 2020;66 (2):183-190.

4. Jacobs SS, Krishnan JA, Lederer DJ, Ghazipura M, Hossain T, Tan AM, et al. Home oxygen therapy for adults with chronic lung disease. An official American Thoracic Society Clinical Practice Guideline. Am J Respir Crit Care Med 2020;202(10):e121-e141.

5. Lacasse Y, Sériès F, Corbeil F, Baltzan M, Paradis B, Simão P, et al. Randomized trial of nocturnal oxygen in chronic obstructive pulmonary disease. N Engl J Med 2020;383(12):1129-1138.

6. The Long Term Oxygen Treatment Trial Research Group, Albert RK, Au DH, Blackford AL, Casaburi R, Cooper JA Jr, et al. A randomized trial of long-term oxygen for COPD with moderate desaturation. $\mathrm{N}$ Engl J Med 2016;375(17):1617-1622. 